\title{
A cross-over study comparing an online versus a paper 7-day food record: focus on total water intake data and participant's perception of the records
}

\author{
B. Monnerie ${ }^{1}$ - L. G. Tavoularis ${ }^{2} \cdot$ I. Guelinckx ${ }^{1} \cdot$ P. Hebel $^{2} \cdot$ T. Boisvieux ${ }^{3}$ \\ A. Cousin $^{4} \cdot$ L. Le Bellego ${ }^{1}$
}

Received: 16 February 2015 / Accepted: 30 May 2015 / Published online: 12 June 2015

(C) The Author(s) 2015. This article is published with open access at Springerlink.com

\begin{abstract}
Purpose To compare (1) fluid, food and nutrient intake obtained with a paper versus an online version of a 7-day food record and (2) user's acceptability of both versions of the food record.

Methods A cross-over study was carried out in 2010 in France. A total of 246 participants aged 18-60 years reported their food and fluid intake using both versions of the 7-day food record, separated by a 7- to 14-day washout period. To help participants in estimating consumed portions, both versions of the food record were supported by a photographic booklet of standard portions and containers. At the end of the study protocol, participants completed a questionnaire designed to assess the acceptability of the two questionnaires. Results The reported water intake of fluids was significantly higher with the online version compared with the paper version (respectively $1348 \pm 36$ and $1219 \pm 34 \mathrm{~mL} /$ day, $p<0.0001$ ). No difference was found between methods in terms of energy intake and the consumption of most food categories, macro- and micronutrients. Furthermore, $77 \%$ of the participants preferred the online method to the paper version.

Conclusions Fluid intake, but not food intake, reported with the online 7-day food record was higher in comparison
\end{abstract}

This article is part of a supplement supported by Danone Nutricia Research.

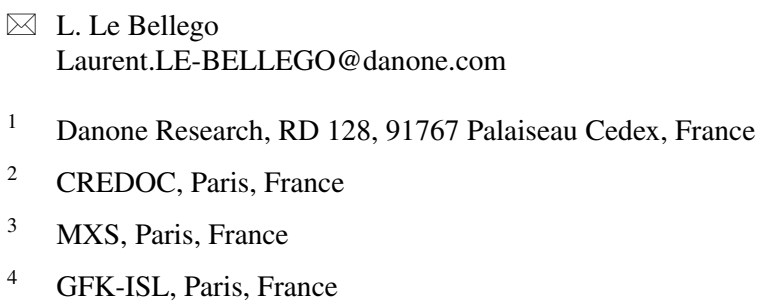

with the paper version. In addition, the online version was preferred by users. In population surveys, the online record is therefore a relevant alternative, and even a preferred alternative in the case of fluid intake, to the paper record.

Keywords Total water intake $\cdot$ Beverages $\cdot$ Fluids . Dietary record $\cdot$ Hydration

\section{Introduction}

Several methods are available to assess individual food and nutrient intake [1]. The accuracy of data collection, however, is often hampered by the acceptability of comprehensive methods that may be perceived by subjects as long and boring [2].

Accurate assessment of fluid intake presents additional problems since fluids are consumed regularly throughout the day and packaging and container (glasses, cans, bottles, carton) size varies greatly. As a result, fluid consumption is rarely reported accurately during dietary surveys. In food studies reporting fluid consumption, there is often a marked difference in intake [3, 4] and the most likely explanation for such discrepancies is the use of different methods of measurement. Further research is therefore needed to accurately measure fluid intake during dietary surveys [4].

Given the need to improve acceptability and reduce the time spent by subjects on dietary surveys, new technologies have been introduced. Since three out of four people in Europe will be using Internet by the end 2014 [5], electronic interfaces are highly relevant and increasingly common, especially for dietary recording in younger generations accustomed to web technologies [6]. A study comparing online and paper versions of a self-administered anthropometric questionnaire highlighted easier data 
Fig. 1 Summary of the study design

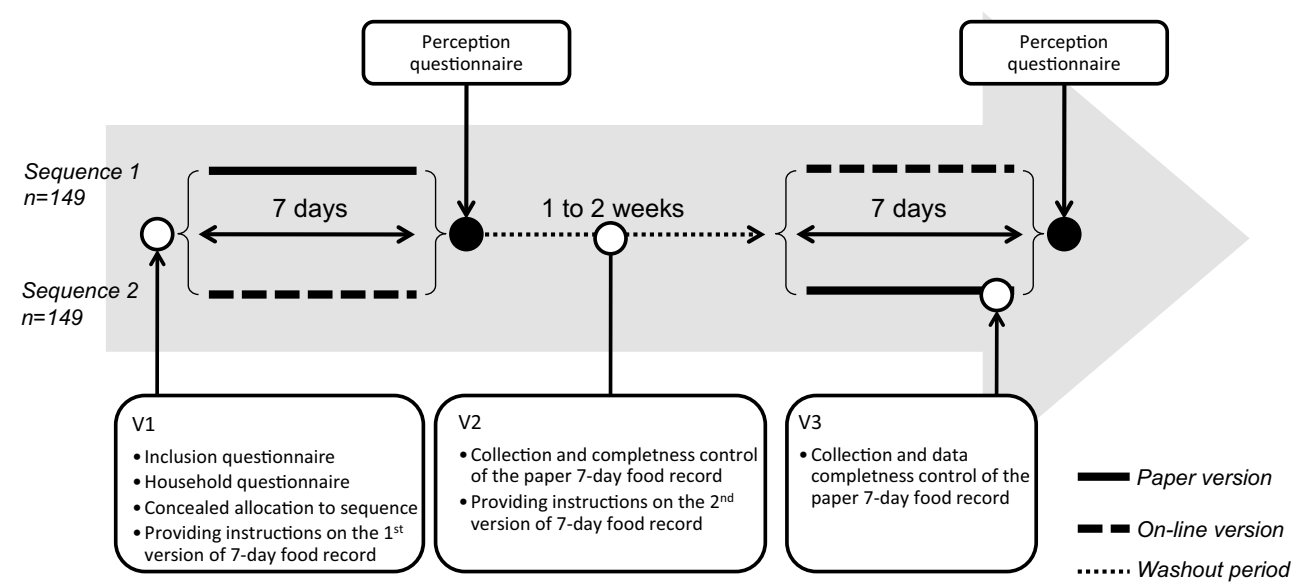

management of the online version [7]. Indeed, data processing appears to be faster and cheaper since data entry by a third party is no longer required. Furthermore, printing, mailing and processing of paper questionnaires are avoided in web-based studies, which considerably impact expenses, paper waste and the environment. Moreover, in case of multicentre or international surveys such as the NutriNetSanté study or HELENA study, online questionnaires have the potential to acquire in a homogenous way the same type of data with a relatively limited amount of resources [8, 9].

However, the method of recording data, online versus on paper, might influence the recording. Therefore, the primary objective of the present study was to compare fluid, food and nutrient data collected using an online 7-day dietary record versus a paper 7-day dietary record. The secondary objective was to compare the acceptability of the two versions of the record by the participants.

\section{Methods}

\section{Study design}

The study was designed as an observational, multicentre, cross-over survey. This cross-over (AB/BA design) study had two periods, one during which the paper version and one during which the online version of the 7-day food record was completed. Both periods were separated by a 7- to 14-day washout period. Participants were alternately upon recruitment allocated to one of the two sequences: sequence 1 started with completing the paper 7-day food record and sequence 2 with the online version. The study design is presented in Fig. 1.

Visit 1 (V1): participants were visited at their home by an interviewer who checked that the inclusion criteria were met. Participants meeting the inclusion criteria were informed about the study objectives and protocol. After signing an informed consent, short instructions were given on the first method of data collection to which they were randomly assigned to (i.e. paper or online version). If a participant was allocated to sequence 1, the paper 7-day food recorded was provided to the participant. If allocated to sequence 2, the participant started with the online 7-day food record. Participants were instructed to keep their usual dietary habits and not to change them through the full protocol. All participants completed a household questionnaire, reporting socio-economic data.

Visit 2 (V2): participants were visited a second time by the interviewer between the two periods. During this visit, instructions on the second method of data collection were given. The paper 7-day food record was also provided to participants of sequence 2 . The interviewers checked with participants of sequence 1 the paper 7-day food record for completeness. All participants completed the perception questionnaire addressing the acceptability of the first version of the 7-day food record.

Visit 3 (V3): participants allocated to sequence 2 received a final visit from the interviewer at the end of the second period. During this visit, interviewers checked the paper 7-day food record for completeness. At the end of the second study period, all participants completed for a second time the perception questionnaire addressing this time the acceptability of the second version of the 7-day food record.

The study protocol was approved by the National Data Protection Authority (Commission Nationale de l'Informatique et des Libertés-CNIL) which oversees ethical issues and protection of individual data collection in France. All the procedures required to render individual data anonymous were performed. Written informed consent was obtained from each participant. However, owing to the descriptive and non-interventional design of this survey, approval by an ethics committee was not required, and the study was not submitted to registration. 


\section{Participants and recruitment}

The inclusion criteria of this study were as follows: having an age between 18 and 60 years, living in France, having a broadband Internet connection at home and being accustomed to using the Internet at least once a month. A quota system was devised to ensure that a broad, nationally representative cross section of households of different composition and type of areas (type/size of home town: rural/ small town/medium-sized city/large city/Paris area) were recruited into the study. Participants were stratified according to sex, age $(<40 / \geq 40$ years old $)$ and professional activity (unemployed/lower socio-professional category/upper socio-professional category).

Interviewers approached individuals at random on the street or they went from door-to-door to recruit participants in order to reduce the selection bias. They continued this random recruitment until all quotas were achieved, and an equal balance between the aforementioned stratification groups was achieved.

\section{Dietary assessment}

The 7-day food record used in this study has been used in large cohort surveys in the past, such as CCAF (Comportements et Consommations Alimentaires en France [10]) and INCA (Enquête Individuelle et Nationale sur les Consommations Alimentaires [3]). The paper 7-day food record was designed to be completed at home, and participants were asked to record all food and fluids consumed during and between meals over the week. It was supplied with a picture-based validated reference book from the SU.VI. MAX survey, which facilitated the assessment of portion size $[11,12]$.

The online version (MXS-Epidemio ${ }^{\circledR}$, Paris, France) was based on the paper 7-day food record and was not used before in a large-scale nutritional survey neither on a period of 7 consecutive days. The software was only accessible online and was not available as an application for a smartphone. The software used a stepwise approach to guide the subject through the completion of the record, following the model of the multiple-pass approach used by the US Department of Agriculture [13]. In a first step, the type of food and fluid consumed at each moment of the day (breakfast, lunch, dinner or any solid or liquid food consumed between meals) was fully registered. In a second step, the portion sizes were entered. To help the subject in the portion size assessment, web screens with calibrated images from the same reference book [12] were shown. The online system performed a match with the corresponding model meal and questioned the user about any forgotten foods. These real-time control procedures helped to minimise the occurrence of missing data. The final validation of data collection was preceded by controls such as the number of food intakes registered, total energy consumption and calculated volume of water intake.

The nutritional content of foods and fluids was calculated based on French food composition tables [14]. Total water intake refers to water from food and fluids; water from fluids refers to the water content of the total drinking water (tap, bottled still, bottled sparkling) plus beverages of all kind (milk, coffee, soft drinks, alcohol, soup, etc.). Fluid intake is the total volume of drinking water and beverages of all kind.

\section{Perception questionnaire}

Acceptability of the two different 7-day food records by the participants was assessed using an ad hoc online questionnaire designed to assess the user's perception on the food records. The questionnaire consisted of 24 questions in total, of which five items were rated on a ten-point scale with a score of ten being the best or most favourable answer. Sixteen items were qualitative questions with a Likert type of scale of four graded answers (not at all, somewhat, rather, very). The questions covered several topics related to the assessment method (e.g. the introduction to the online or paper version, the user-friendliness and the overall impression of the 7-day food records). The mean time spent per day on recording their food and fluid intake was also reported in minutes by the participants.

\section{Criteria for between-methods comparison}

To address the primary objective of the study, several criteria to compare the two version of the 7-day food record were defined in advance. The first criterion was the mean water intake from fluids per day (expressed in $\mathrm{mL} /$ day), and the second criterion was the mean total water intake (i.e. water from fluid and solid food intake) per day. The third criterion was the mean frequency of drinking acts, defined as the number of times per day any fluid type was consumed. The fourth group of criteria was the mean daily energy intake, the mean daily nutrient intake of lipids, carbohydrates (total, simple and complex), proteins, alcohol, fibre, calcium and vitamin D and the frequency of eating acts (one act accounted for each intake of food). The overall score of the perception questionnaire was also used to compare the methods.

\section{Statistical methods}

The statistical model used to compare quantitative variables recorded with both versions of the 7-day record was an analysis of variance (ANOVA) with mixed factors (fixed and random factors). The order of sequence was included 
in the ANOVA model. With this model, the full analysis set (FAS) can be used. The perception questionnaire items was analysed using McNemar's Chi-square test for paired samples. Data management and statistics were performed with SAS 9.2 software. The significance level was set at $p<0.05$. A double-entry procedure was used for the paperbased record. If a dietary record was not completed for at least 4 days, it was considered as missing data.

\section{Results}

\section{Study population}

Overall, 298 participants were recruited into the survey. Among them, 246 participants (i.e. $82.6 \%$ ) completed the dietary assessment for at least 4 days for each method (FAS) and 243 participants completed 7 days for each method, i.e. $81.5 \%$ of the global cohort (per protocol population set).

The cohort was composed of $59 \%$ women. Participants were aged 18-60 years, with 53.5\% aged less than 40 years. More than $80 \%$ used Internet almost every day before the study. There were no significant differences in the demographic characteristics of the participants of both sequences.

\section{Water from fluids and fluid intake}

In the FAS sample, the mean total water intake calculated from data collected with the paper 7-day food record was significantly lower compared to the one from the online record (1812 and $1945 \mathrm{~mL} /$ day, respectively, $p=0.0005$ ). This difference is due to the reporting of fluid intake: the mean daily water from fluid intake calculated from the paper-collected data was significantly lower $(1220 \mathrm{~mL} /$ day) than the one from the online record $(1348 \mathrm{~mL} /$ day; $p=0.0001$ ). The volume of water derived from solid food did not depend on the method of data collection (Fig. 2). Among the different categories of fluids, the difference was statistically significant for still water, sparkling water, still soft drinks, milk and yoghurt drinks (Table 1).

The mean frequency of drinking acts was also significantly lower with the paper record than with the online version of the 7-day food record (Table 1).

\section{Food and nutrient intake}

When comparing the intake of 42 food groups collected with the paper and online dietary record, the intake of only six food groups was significantly different. With the online dietary record, the mean daily intake was significantly higher for desserts $(+23 \% ; p=0.0138)$, pasta $(+21 \%$;

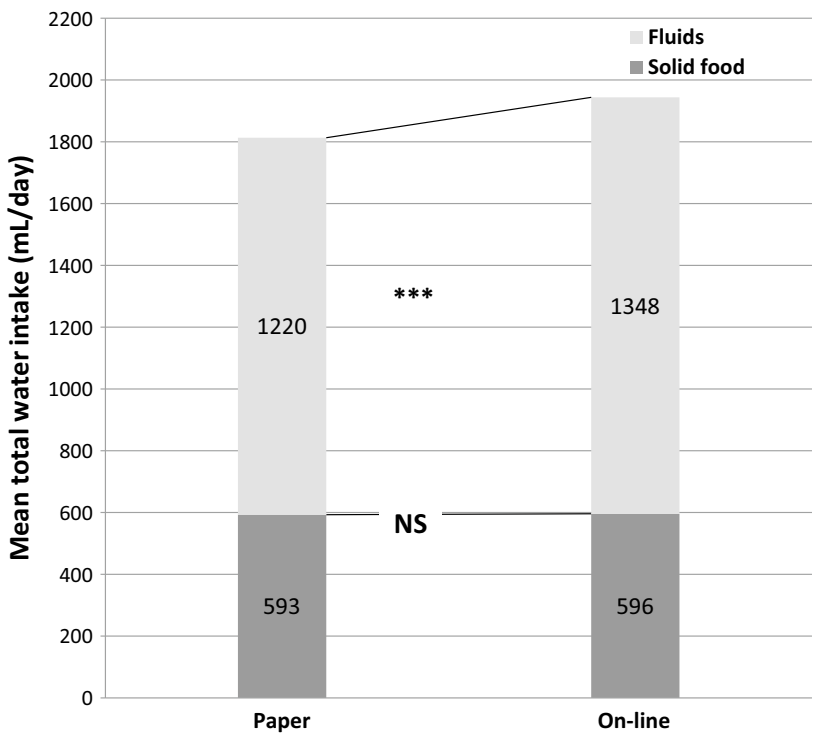

Fig. 2 Mean daily water intake (mL/day) from fluid and solid food. Data are presented as mean $\pm \mathrm{SD}$ and tested with ANOVA. ***Significantly different between paper and online methods $(p<0.0001)$; NS not significantly different

$p=0.0465)$, sweets $(+13 \% ; p=0.0222)$ and condiments $(+21 \% ; p=0.0096)$ and lower for bread and crackers $(-10 \% ; p=0.0231)$ and cooked or processed meat $(-22 \% ; p=0.0023)$ compared to the paper dietary record.

No significant difference was found for energy intake between the paper and the online versions (Table 2). The reported intake of all macro- and micronutrients was comparable between methods, except for simple carbohydrates, calcium and magnesium, which were significantly lower with the paper than with the online version, and for vitamin $\mathrm{D}$ that was higher with the paper version (Table 2).

As for fluid intake, the reported frequency of eating acts was significantly lower with the paper record than with the online version (Table 2). The same trend was observed when considering the total intake (e.g. fluids and solid food together). The difference in frequency of eating and drinking acts between both versions of the record was driven by eating and drinking acts consumed at home (11.6 vs. 13.6 times/day for paper and online methods, respectively, $p<0.0001)$. The number of eating and drinking acts outside the home was similar.

\section{Perception assessment}

A total of 100 participants (40.7\% of the FAS sample) completed the perception questionnaire twice, for example, after using each method of dietary data collection. On average, participants gave significantly higher scores to the online version regarding overall satisfaction with the tool, pleasure in using it and the feeling that the data recorded 
Table 1 Total water intake and intake of different beverages recorded with the paper and online 7-day dietary record

\begin{tabular}{lccr}
\hline & Paper $(n=228)$ & Online $(n=239)$ & $p$ value* \\
\hline Daily volume (mL) of water intake & & \\
Total & $1812 \pm 40$ & $1945 \pm 42$ & 0.0005 \\
From fluids & $1220 \pm 34$ & $1348 \pm 36$ & $<0.0001$ \\
Daily volume (mL)/beverage type & & \\
Still water & $532 \pm 25$ & $610 \pm 29$ & 0.0044 \\
Sparkling water & $44 \pm 9$ & $65 \pm 11$ & 0.0005 \\
Hot drinks & $376 \pm 18$ & $364 \pm 17$ & 0.3481 \\
Alcoholic drinks & $118 \pm 16$ & $105 \pm 11$ & 0.4106 \\
Still soft drinks & $95 \pm 9$ & $99 \pm 10$ & 0.5096 \\
Sparkling soft drinks & $78 \pm 8$ & $94 \pm 11$ & 0.0391 \\
Dairy drinks & $23 \pm 5$ & $42 \pm 6$ & 0.0022 \\
Functional drinks & $0.1 \pm 0.1$ & $1.4 \pm 0.6$ & 0.1219 \\
Daily frequency of & $4.5 \pm 0.1$ & $5.0 \pm 0.1$ & $<0.0001$ \\
$\quad$ drinking acts & & & \\
\hline
\end{tabular}

Values are presented as mean $\pm \mathrm{SD}$

$p$ values less than 0.05 are in italics

* ANOVA with mixed factor with a $p$ value cut-off of 0.05

Table 2 Energy and nutrient intake recorded with the paper and online 7-day dietary record

Paper $(n=228) \quad$ Online $(n=239) \quad p$ value*

\begin{tabular}{|c|c|c|c|}
\hline \multicolumn{4}{|l|}{ Daily energy intake (kcal) } \\
\hline Total & $1836 \pm 41$ & $1825 \pm 39$ & 0.7448 \\
\hline During the week & $1769 \pm 42$ & $1763 \pm 37$ & 0.8695 \\
\hline During the week end & $2005 \pm 52$ & $1983 \pm 52$ & 0.6913 \\
\hline \multicolumn{4}{|c|}{ Daily macronutrient intake (g) } \\
\hline Total fat & $73.8 \pm 2.0$ & $73.2 \pm 1.7$ & 0.7294 \\
\hline Carbohydrates & $199 \pm 5$ & $202 \pm 5$ & 0.5464 \\
\hline Simple carbohydrates & s $81.7 \pm 2.4$ & $87.3 \pm 2.5$ & 0.0033 \\
\hline Proteins & $77.1 \pm 1.7$ & $75.2 \pm 1.5$ & 0.1771 \\
\hline Alcohol & $10.1 \pm 1.2$ & $9.8 \pm 1.2$ & 0.5620 \\
\hline Fibre & $14.7 \pm 0.4$ & $14.9 \pm 0.4$ & 0.6217 \\
\hline \multicolumn{4}{|c|}{ Daily micronutrient intake } \\
\hline Calcium (mg) & $760 \pm 20$ & $802 \pm 18$ & 0.0146 \\
\hline Iron (mg) & $11.3 \pm 0.3$ & $11.2 \pm 0.2$ & 0.7055 \\
\hline Magnesium (mg) & $244 \pm 5$ & $253 \pm 5$ & 0.0396 \\
\hline Sodium (mg) & $2641 \pm 66$ & $2698 \pm 59$ & 0.3477 \\
\hline Vitamin A (IU) & $849 \pm 41$ & $932 \pm 46$ & 0.1108 \\
\hline Vitamin C (mg) & $77.2 \pm 3.3$ & $79.8 \pm 3.1$ & 0.3745 \\
\hline Vitamin D $(\mu \mathrm{g})$ & $2.3 \pm 0.1$ & $2.0 \pm 0.1$ & 0.0210 \\
\hline Vitamin E (mg) & $7.9 \pm 0.3$ & $8.0 \pm 0.2$ & 0.7666 \\
\hline $\begin{array}{l}\text { Frequency of daily } \\
\text { eating acts }\end{array}$ & $10.7 \pm 0.3$ & $12.7 \pm 0.3$ & $<0.0001$ \\
\hline
\end{tabular}

Values are presented as mean $\pm \mathrm{SD}$

$p$ values less than 0.05 are in italics

* ANOVA with mixed factor with a $p$ value cut-off of 0.05 were accurate (Fig. 3). A general linear model indicated that the global perception score for the paper record did not significantly differ according to gender, sex, BMI, professional activity or frequency of Internet use (test Fisher $0.6512 ; p>0.05)$. For the online record, only BMI but none of the other factors had a significant effect on the global perception score (test Fisher 0.048; $p<0.05$ ). For both methods, the instructions received before starting data collection was perceived as satisfactory (score 7.9/10), useful (for $95 \%$ of participants) and the information given to assess food intake was considered clear (for $95 \%$ of participants). However, more participants considered the general layout and the graphical user interface as clear and friendly with the online version (Table 3). The online version was also assessed as more convenient, quicker and easier than the paper version (Table 3). Participants spent significantly more time completing the record using the paper version compared to the online version (34.4 vs. $28.5 \mathrm{~min} / \mathrm{day}$, $p=0.045$ ). Participants also appeared to master the completion of the online record faster than the paper record (Table 3).

Finally, in response to the question "Which method did you prefer?", $77.7 \%$ of the participants who completed both methods preferred the online version, $13.2 \%$ preferred the paper version, and $9.1 \%$ did not report any preference.

\section{Discussion}

The present study aimed to compare fluid, food and nutrient intake obtained with a paper versus an online version of a 7-day food record. To our knowledge, there is only one recently published pilot study that compare different dietary survey methods with a specific focus on the assessment of water from fluids [15]. This specific focus for fluid intake aimed to test our hypothesis that fluid intake is underestimated while using traditional paper food records. The results of the current study tend to support this hypothesis: total water intake, water from fluids and the reported volume of drinking water and other beverages types calculated from the online 7-day record were significantly higher compared to those calculated from the paper 7-day record. A possible explanation for these differences is the omission of drinking acts taking place outside meals. This explanation is derived from an observation made during a crosssectional survey specifically assessing fluid intake where drinking acts took place not only during meals such as food intake, but also outside meal and this throughout the day [16]. The online interface was therefore designed to prevent fluids being forgotten during recording, especially between meals. An automatic reminder (contextual menu or pop-up window) appeared before entering each main meal, which was not available in the paper version. Indeed, the results 
Fig. 3 Mean acceptability scores based on the results of the perception questionnaire for the online and the paper 7-day dietary record. $N S$ not significantly different. Participants rated each item from 0 (not at all) to 10 (totally)

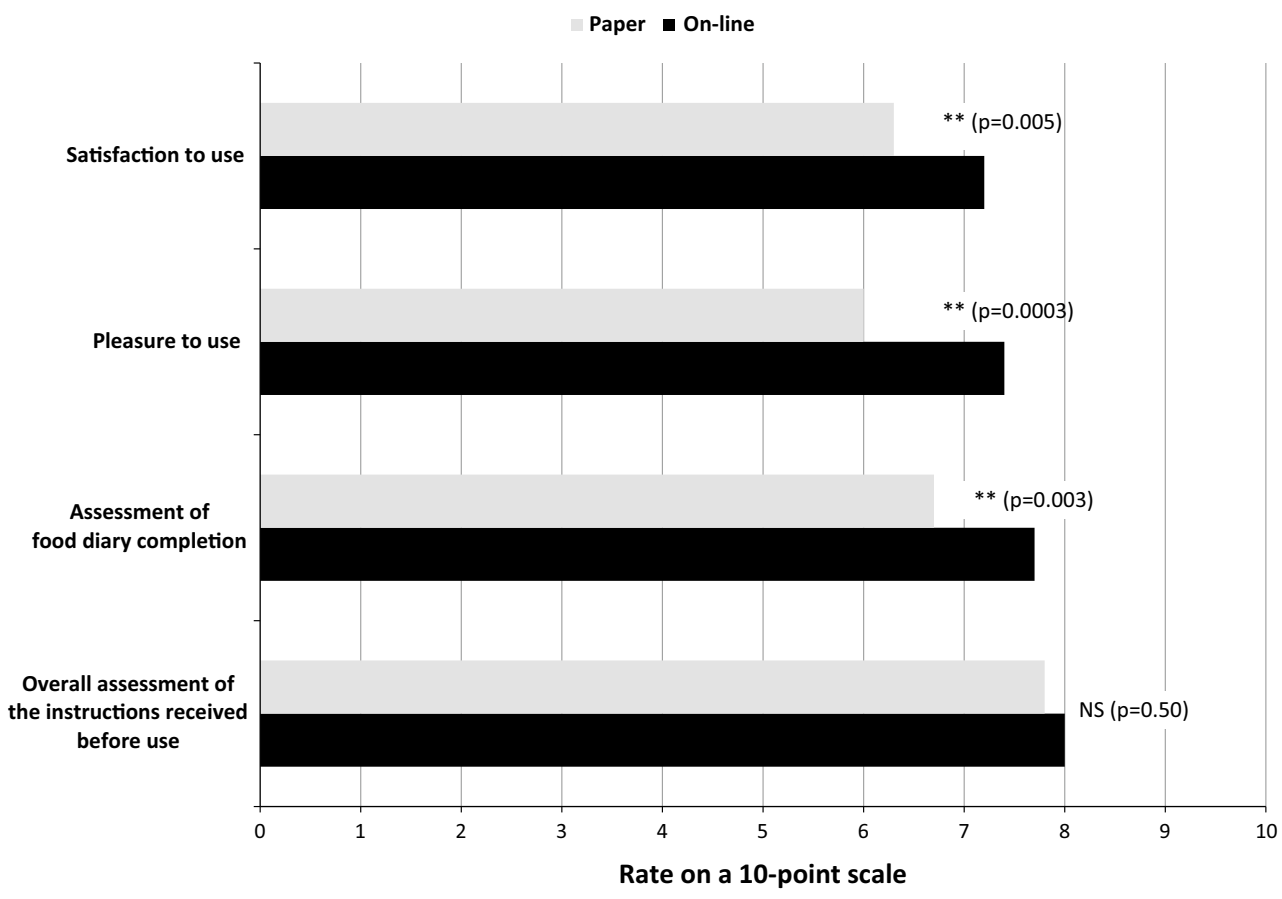

Table 3 Participants' perception of the online and the paper version of the 7-day dietary record

\begin{tabular}{|c|c|c|c|}
\hline & Paper $(\%)$ & Online $(\%)$ & $p$ value \\
\hline \multicolumn{4}{|c|}{ Appreciation of the general layout of the questionnaire } \\
\hline Clear $^{\mathrm{a}}$ & 76.1 & 95.9 & $\leq 0.0001^{*}$ \\
\hline Friendly $^{\mathrm{a}}$ & 83.1 & 94.3 & $0.0018 *$ \\
\hline \multicolumn{4}{|c|}{ Perception of the data collection tool } \\
\hline Convenient $^{\mathrm{a}}$ & 62.3 & 89.4 & $\leq 0.0001 *$ \\
\hline Quick $^{\mathrm{a}}$ & 51.6 & 85.2 & $\leq 0.0001 *$ \\
\hline Easy $^{\mathrm{a}}$ & 70.8 & 91.0 & $\leq 0.0001 *$ \\
\hline
\end{tabular}

Number of days needed to master to the completion of the method

\begin{tabular}{lrrr} 
From day $1^{\mathrm{b}}$ & 20.0 & 34.4 & $0.0009 *$ \\
From day $2^{\mathrm{b}}$ & 37.7 & 48.4 & \\
From day $3^{\mathrm{b}}$ & 31.5 & 13.1 & \\
${\text { Beyond day } 4^{\mathrm{b}}}^{\mathrm{N}}$ & 6.9 & 3.3 \\
Never $^{\mathrm{b}}$ & 3.8 & 0.8 & \\
\hline
\end{tabular}

$p$ values less than 0.05 are in italics

a Expressed as the percentage of participants who qualified the method "very" or "rather"

${ }^{b}$ Expressed as the percentage of participants who declared mastering the completion of the 7-day dietary record

* McNemar's Chi-square test for paired sample

suggest that the interactive interface did help participants to remember fluids consumed: the frequency of drinking acts declared with the online method was higher than with the paper record. On the other hand, in the absence of a referential method such as a biomarker, one cannot conclude that the data obtained with the online paper is more accurate.
These automatic reminders prompting subjects to revise their reported intakes might lead to an over-reporting, in particular, for foods considered as healthy. Nevertheless, combining the observations of this cross-over trial and the cross-sectional survey discussed earlier, the assumption can be made that the online record more accurately reflects the actual fluid intake of participants. A practical key learning to retain from this finding is that whenever a participant is requested to record their food and fluid intake, extra questions regarding water and beverages intake within and outside meals should be raised.

As for the daily frequency of drinking acts, the daily frequency of eating acts was also higher with the online version of the record. Yet, the total daily energy intake and amounts of solid food were not statistically different between the two methods. These results are consistent with those obtained comparing online and paper versions of a multiple 24-h recall questionnaire [17]. Examining the intake of different food categories collected with the two versions of the dietary record, results showed no significantly difference for the 42 considered food categories, except for six groups. These differences in these few food categories are difficult to interpret and should be confirmed in future experiments that include biomarkers.

Another observation worth to comment relates to responders fatigue over the 7-day recording period. The statistical analysis tested the effect of the day of recording on the energy intake reported using a covariance model (data not shown), which could indicate the participant's fatigue over time. With the online record, the effect of the day of recording is absent and energy intake remained 
constant over the week of reporting, whereas it decreased with the paper version. This observation suggests that using the online record reduces respondents fatigue over time.

These data are congruent with the evaluation of perception. Indeed, $44.6 \%$ of the participants thought that they had not forgotten any food intake with the paper tool versus $57.4 \%$ with the online tool. Conversely, $20.8 \%$ of the participants using the paper tool felt that they had forgotten a lot of details during their recording versus $11.5 \%$ with the online tool. These results provide support for the online tool in terms of the completeness of data recording. An addition argument in favour of the online tool is the fact that most participants declared that they preferred the online version of the questionnaire. This is consistent with another French study comparing a self-administered, online and paper-based socio-demographic and economic questionnaire. In all, $93.7 \%$ of their participants preferred the online version. Substantial logistic and cost advantages were also demonstrated [8]. Against expectations, the perception scores for both tools were not different according to age, gender, professional activity or the frequency of Internet use. This lack of significant difference in perception score might, however, be due to the limited sample size of the stratified groups.

Despite the advantages listed above of an online questionnaire, the differential access to the Internet may raise concerns. Online questionnaires could exclude some populations such as elderly people and disadvantaged social classes [18]. However, Internet access is continually growing and may not be a real limitation in the near future [7]. In France, $64 \%$ of homes subscribed to an Internet connection in 2011 versus $12 \%$ in 2001 [19]. In the present study, the stratification of participants according to gender, age, socio-economic status and home environment aimed to prevent this selection bias.

To conclude, this study comparing two versions of a 7-day food questionnaire showed that an online record captured more drinking and eating acts than a paper version. Consequently, the online version resulted in a significantly higher mean intake of water from beverages and drinking water, despite the comparable or small differences between the two methods in terms of energy intake, and macroand micronutrient intake. Even though a future confirmation with biomarkers is needed, these findings suggest that the online record more accurately reflects the actual daily fluid intake. Moreover, the online record was rated to be highly acceptable and user friendly and reduced the participants' time spent on recording their intakes. These findings highlight the opportunity for the use of an online 7-day record as a medium to record fluid intake of large-scale populations.
Acknowledgments The authors wish to thank Jean-François Boisvieux (MXS), Françoise Montillet (GfK-ISL), Bridget Holmes (Danone Research) and Isabelle Boutrolle (Danone Research) for their contribution to this work.

Conflict of interest The study was funded by Danone Research. M.B., G.I. and L.B.L. are employees of Danone Research. B.T. is an employee of MXS, the company in charge of developing the online version of the 7-day food record. T.L.G. and H.P. are employees of CREDOC, the organization performing the data collection. C.A. has no conflict of interest to declare.

Ethical standards The study protocol was approved by the National Data Protection Authority (Commission Nationale de l'Informatique et des Libertés-CNIL) which oversees ethical issues and protection of individual data collection in France. All the procedures required to render individual data anonymous were performed. Detailed information about the study and its objectives, what was expected from them, as well as a disclosure of the study's provisions to preserve confidentiality, risks and benefits, and a clear explanation about their option to participate voluntarily or not in the study was provided to each participant. After offering this full-informed description of the study, following the principles of informed consent, written informed consent was obtained from each participant. However, owing to the descriptive and non-interventional design of this survey, approval by an ethics committee was not required, and the study was not submitted to registration.

Open Access This article is distributed under the terms of the Creative Commons Attribution 4.0 International License (http://creativecommons.org/licenses/by/4.0/), which permits unrestricted use, distribution, and reproduction in any medium, provided you give appropriate credit to the original author(s) and the source, provide a link to the Creative Commons license, and indicate if changes were made.

\section{References}

1. Bates C, Nelson M, Holmes B, Ulijaszek S (2010) Nutritional assessment methods. In: Geissler CPH (ed) Human nutrition, 12th edn. Elsevier, Amsterdam

2. Thompson F, Subar A (2008) Dietary assessment methodology. In: Coulston AMBC (ed) Nutrition in the prevention and treatment of disease, 2nd edn. Academic Press, San Diego

3. Dubuisson C, Lioret S, Touvier M, Dufour A, Calamassi-Tran $G$ et al (2010) Trends in food and nutritional intakes of French adults from 1999 to 2007: results from the INCA surveys. Br J Nutr 103:1035-1048

4. Bellisle F, Thornton SN, Hebel P, Denizeau M, Tahiri M (2010) A study of fluid intake from beverages in a sample of healthy French children, adolescents and adults. Eur J Clin Nutr 64:350-355

5. ICT Data and Statistics Division, Telecommunication Development Bureau (2014) Mobile-broadband uptake continues to grow at double-digit rates. http://www.itu.int/en/ITU-D/Statistics/Documents/facts/ICTFactsFigures2014-e.pdf. Accessed 12 Jan 2015

6. Boushey CJ, Kerr DA, Wright J, Lutes KD, Ebert DS et al (2009) Use of technology in children's dietary assessment. Eur J Clin Nutr 63(Suppl 1):S50-57

7. Touvier M, Mejean C, Kesse-Guyot E, Pollet C, Malon A et al (2010) Comparison between web-based and paper versions of a self-administered anthropometric questionnaire. Eur J Epidemiol 25:287-296 
8. Vergnaud AC, Touvier M, Mejean C, Kesse-Guyot E, Pollet C et al (2011) Agreement between web-based and paper versions of a socio-demographic questionnaire in the NutriNet-Sante study. Int J Public Health 56:407-417

9. Vereecken CA, Covents M, Sichert-Hellert W, Alvira JM, Le Donne C et al (2008) Development and evaluation of a selfadministered computerized 24-h dietary recall method for adolescents in Europe. Int J Obes 32(Suppl 5):S26-34

10. Hébel P (2007) Comportements et consommations alimentaires en France. Lavoisier, Paris

11. Hercberg S, Preziosi P, Briancon S, Galan P, Triol I et al (1998) A primary prevention trial using nutritional doses of antioxidant vitamins and minerals in cardiovascular diseases and cancers in a general population: the SU.VI.MAX study-design, methods, and participant characteristics. SUpplementation en VItamines et Mineraux AntioXydants. Control Clin Trials 19:336-351

12. SUVIMAX (2000) Portions alimentaires: manuel photos pour l'estimation des quantités. Edtitions Polytechnica, Paris

13. Raper N, Perloff B, Ingwersen L, Steinfeldt L, Anand J (2004) An overview of USDA's dietary intake data system. J Food Compos Anal 17:545-555
14. Favier J-C, Ireland-Ripert J, Toque C, Feinberg M (1995) CIQUAL Répertoire général des aliments-Table de composition. Lavoisier, Paris

15. Nissensohn M, Lopez-Ufano M, Castro-Quezada I, Serra-Majem L (2015) Assessment of beverage intake and hydration status. Nutr Hosp 31(Suppl 3):62-69

16. Gandy J (2010) First findings of the United Kingdom fluid intake study. Nutr Today 47(4S):S14-S16

17. Beasley JM, Davis A, Riley WT (2009) Evaluation of a webbased, pictorial diet history questionnaire. Public Health Nutr 12:651-659

18. Eysenbach G (2004) Improving the quality of Web surveys: the Checklist for Reporting Results of Internet E-Surveys (CHERRIES). J Med Internet Res 6:e34

19. Gombault V (2011) Deux ménages sur trois disposent d'internet chez eux. INSEE Première 1340:1-4 